\title{
Long-term efficacy and safety of lamotrigine for all types of bipolar disorder
}

This article was published in the following Dove Press journal:

Neuropsychiatric Disease and Treatment

20 March 2017

Number of times this article has been viewed

\author{
Yoshinori Watanabe ${ }^{1,2}$ \\ Seiji Hongo ${ }^{2}$ \\ 'Himorogi Psychiatric Institute, Tokyo, \\ ${ }^{2}$ Nanko Clinic of Psychiatry, Shirakawa \\ city, Fukushima, Japan
}

Background: We investigated whether the long-term efficacy and safety of lamotrigine (LTG) for bipolar disorder (BP) differs between disease types (BP-I, BP-II, or BP not otherwise specified [BP-NOS]), and the efficacy of the concomitant use of antidepressants (ADs).

Methods: For $>1$ year, we observed 445 outpatients with BP (diagnosed by DSM-IV criteria) who initiated LTG treatment between July 1 and October 31, 2011, using the Himorogi Selfrating Depression (HSDS) and Anxiety Scales and the Clinical Global Impression-Improvement scale and also recorded adverse events.

Results: Treatment efficacy was observed at week 4, with the improved HSDS scores sustained until week 52 for all types of BP; $50 \%$ of the patients with any type of BP could be treated with LTG for 1 year, whereas $\sim 40 \%$ could be treated for $>1.5$ years. However, $25 \%$ of the patients were withdrawn within the first 4 weeks. The overall incidence of adverse events was 22.9\% (104/455): $34.1 \%$ (14/41) for BP-I, $22.7 \%$ (15/66) for BP-II, and 22.2\% (75/338) for BP-NOS. The most common adverse event was skin rash: $22.0 \%$ for BP-I, $16.7 \%$ for BP-II, and $12.1 \%$ for BP-NOS.

Limitations: There was no control group. Data were collected retrospectively.

Conclusion: With careful and adequate titration, long-term treatment with LTG is possible for any type of BP, with BP-NOS patients, the largest population in clinical practice, responding particularly well. Symptoms can improve with or without ADs. Large-scale prospective studies of the efficacy of ADs in bipolar treatment are warranted.

Keywords: lamotrigine, bipolar disorder, long-term efficacy, antidepressant, skin rash, anxiety

\section{Introduction}

Bipolar disorder (BP) is an illness characterized by recurrent episodes of depression and mania (bipolar I disorder; BP-I) or hypomania (bipolar II disorder; BP-II). ${ }^{1}$ According to a worldwide survey on mental health, the prevalence of BPs was consistent across diverse cultures with a lifetime prevalence of $0.6 \%$ for BP-I, $0.4 \%$ for BP-II, $1.4 \%$ for subthreshold BP, and $2.4 \%$ for the BP not otherwise specified (BP-NOS). ${ }^{2}$

Almost half of the episodes of BP present initially with depression, ${ }^{3}$ and depressive symptoms tend to last far longer than periods of mania or hypomania, ${ }^{4,5}$ which may explain why antidepressants (ADs) remain the most prevalent class of psychotropic drug treatment administered to patients with BPs. ${ }^{6-8}$ However, it has been suggested that ADs may increase the risk of manic induction and cycle acceleration. ${ }^{9-11}$ Furthermore, both anxiety disorder comorbidity, which has a lifetime prevalence of $45 \%$ in individuals with BPs, ${ }^{12}$ and exposure to a greater number of AD trials have been suggested as independent predictors of prospective long-term nonresponse to treatment. ${ }^{13}$ In addition, a recent study on predictors of nonadherence among
Correspondence: Yoshinori Watanabe Himorogi Psychiatric Institute, Ichigaya-Asukara Bldg. IF-2F, 2-3I-3 Ichigayatamachi, Shinjuku-ku, Tokyo 162-0843, Japan

Tel $+8 I 359468586$

Fax +8I 359468587

Email watanabe@himorogi.org
Neuropsychiatric Disease and Treatment 2017:13 843-854

843 
patients with mood disorders reveals that substance use disorder and illness severity are significant predictors of nonadherence especially in patients with $\mathrm{BP}$, whereas treatment side effects are of primary importance for depressive disorder. ${ }^{14}$

Poor adherence to medication has also been reported to have an impact on clinical and safety outcomes of patients with BPs. ${ }^{15,16}$

Lamotrigine (LTG) is a widely used antiepileptic drug that is also effective in the treatment of BP, particularly in the prevention of depressive episodes. ${ }^{17,18}$ In the USA and the European Union, LTG is licensed for the prevention of relapse in patients with BP-I who have predominantly depressive episodes. ${ }^{19}$ In Japan, it was approved in July 2011 as the first drug to prevent the recurrence/relapse of mood episodes in patients with BP. ${ }^{20}$ However, skin rash remains a particular concern with LTG treatment, with an incidence of $8 \%-11 \%,{ }^{21}$ although the rate of severe rashes has declined from $1 \%$ to $0.1 \%-0.01 \%$ since the introduction of a gradual titration schedule in $1994 .{ }^{22}$

Most of the large-scale clinical trials investigating the efficacy and tolerability of pharmacological treatments in patients with BP have been conducted for BP-I. Of the 33 known trials, 15 (45\%) were for BP-I, 4 (12\%) for BP-II, 8 (24\%) for both BP-I and BP-II, and 6 (18\%) for BP-NOS. ${ }^{23}$ The only trial investigating the efficacy of long-term treatment of LTG in patients with BP in Japan has been a doubleblind controlled study for BP-I conducted across 60 sites. ${ }^{20} \mathrm{In}$ this study, we examined the safety and efficacy of long-term LTG treatment in patients with BP-I and BP-II, as well as in patients with BP-NOS, as the majority of patients with BP in real-world clinical settings are BP-NOS, rather than BP-I. We also investigated the efficacy of the concomitant use of ADs with LTG in the treatment of BP within a long-term observational setting.

\section{Methods}

\section{Study design and participants}

A total of 445 outpatients who initiated LTG treatment between July 1 and October 31, 2011, at Himorogi Psychiatric Institute (Tokyo) or Nanko Clinic of Pschiatry (Fukushima) were included. Prior to the study, the protocol was reviewed and approved by the institutional review board of Himorogi Psychiatric Institute. The purpose and methods of the study were explained to all patients and written informed consent was obtained. Medical records of the patients were evaluated from the initiation of treatment until week 52 or withdrawal. All data were obtained when patients attended their psychiatric examinations and/or treatment.
The inclusion criteria were as follows: 1) a diagnosis of BP as defined by DSM-IV-TR; 2) initiation of LTG treatment between July 1 and October 31, 2011, at one of the participating sites; and 3) at least 20 years of age. Patients were excluded if 1) they had a known hypersensitivity to LTG ingredients; 2) they deviated from the dosage and administration descriptions in the LTG package; or 3) they were rediagnosed with a disease other than BP between July 1 and October 31, 2011.

\section{Measures}

The following information was collected from the medical records of eligible patients: 1) patient information (age at initial visit, sex, concomitant medications, and age at onset); 2) LTG treatment (date of initiation, age at initiation, initial dose, maintenance dose, and disease type at initiation [BP-I, BP-II, or BP-NOS]); 3) withdrawal from LTG (date of withdrawal), treatment duration (dates of withdrawal/initiation), and reasons for withdrawal; 4) efficacy, based on a number of measures described below; and 5) adverse events, as a measure of safety.

Medication efficacy was assessed through changes in psychological instrument scores and in the medications administered. Scores for the Himorogi Self-rating Depression Scale (HSDS) ${ }^{24}$ and Himorogi Self-rating Anxiety Scale $(\text { HSAS })^{25}$ were collected for weeks 4, 8, 12, 24, 36, and 52 after the initiation of LTG. The score for the Clinical Global Impression Improvement (CGI-I) ${ }^{26}$ scale at week 52 was also recorded. In addition, concomitant medications for mental disorder were noted, including psychotropic agents, atypical antipsychotic drugs, ADs, selective serotonin reuptake inhibitors (SSRIs), serotonin norepinephrine reuptake inhibitors (SNRIs), and tricyclic ADs (TAs).

HSDS and HSAS were both developed by Himorogi Psychiatric Institute with the aim of making the evaluation of items in depression and anxiety scales that had been developed for Western culture applicable to Japanese culture. Both are 10-item self-reported scales with scores ranging from 0 to 39 , with lower scores indicating milder symptoms. Mimura et $\mathrm{a}^{24,25}$ have suggested that HSDS and HSAS are good substitutes for the Hamilton Rating Scale for Depression/ Anxiety, ${ }^{27,28}$ the most commonly used depression/anxiety scales in clinical studies outside Japan. ${ }^{29}$ Cronbach's alpha coefficients for the HSDS were 0.85 (95\% confidence interval [CI]: $0.82-0.88),{ }^{24}$ and those for the HSAS were $0.87(95 \%$ CI: $0.85-0.90){ }^{25}$

The CGI severity and improvement scales describe a patient's overall clinical state as a "global impression" by the rater, providing overall clinician-determined summary 
measure that takes into account all available information, including the patient's history, psychosocial circumstances, symptoms, etc. ${ }^{30}$ The CGI has been shown to correlate well with standard, well-known research drug efficacy scales, such as HAM-D, across a wide range of psychiatric indications. ${ }^{31-33}$ The raters of CGI for the study were the authors of this study who had meetings several times to discuss and confirm the improvement of each patient by referring to the fluctuations of the HSDS/HSAS scores and other available information on the patients.

The primary endpoint of this study was the change in HSDS score from baseline to week 52 or to withdrawal after the LTG treatment. The secondary endpoints were the changes in HSDS and HSAS scores at weeks 4, 8, 12, 36, and 52 after initiation of the LTG treatment, CGI-I score at week 52, and adverse events reported during the study period, with their incidence.

\section{Statistical analysis}

SPSS Statistics 17.0 was used for the statistical analysis. Continuous data were summarized as mean \pm standard deviation, with the number of patients and discrete data summarized as frequencies (\%). Adherence to LTG treatment was analyzed using the Kaplan-Meier method with medians of treatment period (days); this method also used additional data that exceeded 1 year (52 weeks) after LTG initiation. Time course changes in HSDS/HSAS scores were analyzed by paired $t$-tests using paired data that were available at both the evaluation points and baseline. The level of statistical significance was set as $\alpha=0.05$, two-sided, unless otherwise specified. Missing values were not supplemented and only the actual observation data were used.

\section{Results}

\section{Baseline characteristics of the patients}

A total of 445 patients initiated LTG treatment between July 1 and October 31, 2011: 41 (9.2\%) with BP-I, 66 (14.8\%) with BP-II, and 338 (76\%) with BP-NOS. Table 1 presents further characteristics of the patients. Nearly $60 \%$ of the patients were females; this was also the case for each disease group. The mean age of all patients at initial visit was $34.8 \pm 11.2$ years; however, the age for the BP-I

Table I Patient background and baseline characteristics

\begin{tabular}{|c|c|c|c|c|}
\hline Characteristic & All $(n=445)$ & BP-I $(n=4 I)$ & BP-II $(n=66)$ & BP-NOS $(n=338)$ \\
\hline Male: $\mathrm{n}(\%)$ & $177(39.8)$ & $18(43.9)$ & $24(36.4)$ & $135(39.9)$ \\
\hline Age at initial visit: mean $\pm S D$ & $34.8 \pm \mid 1.21$ & $39.7 \pm 14.62$ & $35.5 \pm 10.95$ & $34.1 \pm 10.66$ \\
\hline Age at onset: mean $\pm S D$ & $29.1 \pm 10.60$ & $31.4 \pm 13.98$ & $29.9 \pm 10.19$ & $28.7 \pm 10.19$ \\
\hline \multicolumn{5}{|c|}{ Depression/anxiety scores at baseline: mean \pm SD } \\
\hline HSDS & $21.1 \pm 7.16(430)$ & $17.6 \pm 7.12(38)$ & $22.1 \pm 6.05(66)$ & $21.3 \pm 7.27(326)$ \\
\hline HSAS & $21.3 \pm 9.83(410)$ & $18.0 \pm 9.90(30)$ & $21.6 \pm 8.86(60)$ & $21.6 \pm 9.97(320)$ \\
\hline \multicolumn{5}{|l|}{ LTG initial daily dose (mg): $n(\%)$} \\
\hline 5 & $19(4.3)$ & $\mathrm{I}(2.4)$ & $4(6.1)$ & $14(4.1)$ \\
\hline 12.5 alternate days & $5(1.1)$ & 0 & 0 & $5(1.5)$ \\
\hline 12.5 & $42(9.4)$ & $2(4.9)$ & $5(7.6)$ & $35(10.4)$ \\
\hline 25 alternate days & $127(28.5)$ & $10(24.4)$ & $25(37.9)$ & $92(27.2)$ \\
\hline 25 & $246(55.3)$ & $26(63.4)$ & $30(45.5)$ & $190(56.2)$ \\
\hline 50 & $3(0.7)$ & $\mathrm{I}(2.4)$ & $2(3.0)$ & 0 \\
\hline 100 & $\mathrm{I}(0.2)$ & 0 & 0 & $\mathrm{I}(0.3)$ \\
\hline Unknown & $2(0.4)$ & I (2.4) & 0 & I $(0.3)$ \\
\hline \multicolumn{5}{|c|}{ LTG daily maintenance dose (mg): $n$ (\%) } \\
\hline$<5.0$ & $2(0.4)$ & 0 & 0 & $2(0.6)$ \\
\hline $5.0-12.4$ & $10(2.2)$ & I (2.4) & 0 & $9(2.7)$ \\
\hline $12.5-24.9$ & $21(4.7)$ & 0 & $\mathrm{I}(\mathrm{I} .5)$ & $20(5.9)$ \\
\hline $25.0-49.9$ & $32(7.2)$ & $3(7.3)$ & $4(7.6)$ & $25(7.4)$ \\
\hline $50.0-99.9$ & $55(12.4)$ & $6(14.6)$ & $7(10.6)$ & $42(12.4)$ \\
\hline $100.0-199.9$ & $61(13.7)$ & $7(17.1)$ & $9(13.6)$ & $45(13.3)$ \\
\hline $200.0-400.0$ & $54(12.1)$ & $8(19.5)$ & $10(15.2)$ & $36(10.7)$ \\
\hline Withdrawal: n (\%) & $210(47.2)$ & $16(39.0)$ & $35(53.0)$ & $159(47.0)$ \\
\hline LTG duration (day): mean \pm SD & $176.1 \pm 223.22$ & $322.6 \pm 397.54$ & $218.7 \pm 290.88$ & $150.1 \pm 164.08$ \\
\hline \multicolumn{5}{|l|}{ Concomitant drugs: n (\%) } \\
\hline Psychotropics & $345(77.5)$ & $35(85.4)$ & $60(90.9)$ & $250(74.0)$ \\
\hline Atypical antipsychotics & $125(28.1)$ & $17(4 \mid .5)$ & $22(33.3)$ & $86(25.4)$ \\
\hline ADs & $216(48.5)$ & $14(34.1)$ & $39(59.1)$ & $163(48.2)$ \\
\hline
\end{tabular}

Abbreviations: ADs, antidepressants; BP-I, bipolar I disorder; BP-II, bipolar II disorder; BP-NOS, bipolar disorder not otherwise specified; HSAS, Himorogi Self-rating Anxiety Scale; HSDS, Himorogi Self-rating Depression Scale; LTG, lamotrigine; SD, standard deviation. 
group was $39.7 \pm 14.6$ years, which was older than for the other groups. Similarly, the mean age at onset overall was $29.1 \pm 10.6$ years, but $31.4 \pm 14.0$ years for the BP-I group. The mean HSDS score at baseline for all the patients was 21.1 $\pm 7.16(\mathrm{n}=430)$, with BP-II showing the worst score of $22.1 \pm 6.05$, followed by $21.3 \pm 7.3(\mathrm{n}=326)$ for BP-NOS and 17.6 \pm 7.1 for BP-I. The HSAS scores at baseline followed a similar pattern to the HSDS scores. As the initial daily dose, $25 \mathrm{mg}$ was administered to $\sim 50 \%$ of the patients, with $\sim 30 \%$ receiving $25 \mathrm{mg}$ on alternate days and $\sim 10 \%$ receiving $12.5 \mathrm{mg}$. The BP-NOS group showed the shortest treatment duration (150.1 \pm 164.1 days), and BP-I the longest (322.6 \pm 397.5 days). The overall withdrawal rate was $47.2 \%$, with rates of $39.0 \%, 53.0 \%$, and $47.0 \%$ for BP-I, BP-II, and BP-NOS, respectively, for various reasons. The highest approved maintenance dose of $400 \mathrm{mg}$ was found in BP-I (19.5\%) and BP-II (15.2\%), and the second highest dose of $200 \mathrm{mg}$ was found in BP-NOS (13.3\%).

The rate of concomitant medication with psychotropic drugs was $77.5 \%$ (345/445); rates for BP-I (85.4\%: 35/41) and BP-II (90.9\%: 60/66) were higher than for BP-NOS (74.0\%: 250/338). Nearly half of the patients (48.5\%: 216/445) were on multiple drug treatment with ADs, and nearly $30 \%$ of the patients $(28.1 \%: 125 / 445)$ were treated concomitantly with atypical antipsychotics.

The average number of medications for the 345 patients on multidrug therapy was 3.54 psychotropic drugs: 1.21 atypical antipsychotics for 125 patients and $1.46 \mathrm{AD}$ for 216 patients (Table 2A). These numbers of medications are also indicated as doses in Table 2B. Doses of ADs including SSRI, SNRI, and TA were equivalently converted into those of imipramine, and those of atypical antipsychotics were converted into those of chlorpromazine. However, since there was no mutually standard drug among all the classes of psychotropic drugs, the mean doses for the total psychotropics could not be calculated. As shown by the classes of psychotropic drugs according to disease type, the BP-II group received the lowest number of drugs in both atypical antipsychotics (1.09 vs total: 1.21) and ADs (1.33 vs 1.46 for all patients). TAs were used more commonly with BP-I (1.50) and BP-II (1.43) than with BP-NOS (1.08).

\section{Changes in HSDS and HSAS scores}

Time course changes in the HSDS and HSAS scores were analyzed where paired data were available at baseline and at weeks 24 and 52. At week 24, the trend of improvement (ie, the decrease in HSDS scores) slowed and stabilized, with reduced mean scores of $4.54(-24.10 \%: 21.1-16.5, P<0.001)$

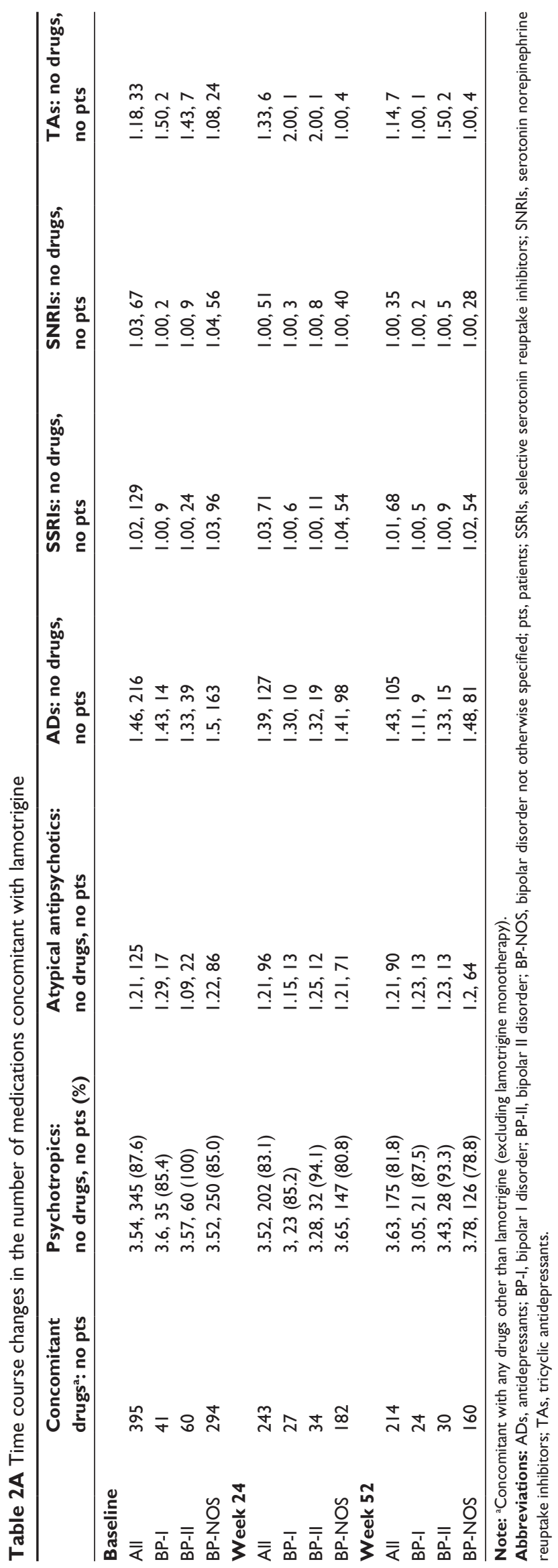




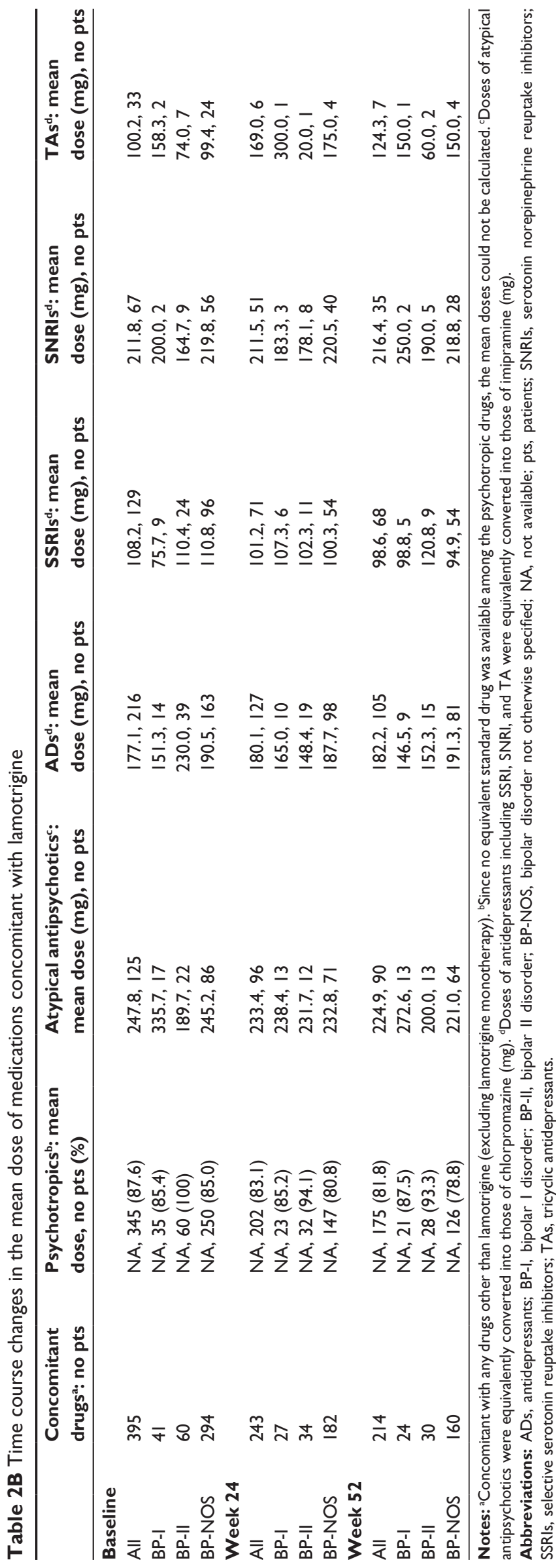

for all patients, $3.59(-20.40 \%$ : 17.6-14.0, $P=0.285)$ for BP-I, $7.64(-34.57 \%$ : 22.1-14.5, $P<0.001)$ for BP-II, and 3.98 (-18.69\%: 21.3-17.3, $P<0.001)$ for BP-NOS. At week 52, the decrease in HSDS scores was sustained, with reduced mean scores of 5.64 (-26.73\%: 21.1-15.4, $P<0.001)$ for all patients, $4.03(-22.90 \%$ : 17.6-13.5, $P=0.210)$ for BP-I, $7.98(-36.11 \%: 22.1-14.1, P<0.001)$ for BP-II, and 5.33 $(-25.02 \%$ : 21.3-16.0, $P<0.001)$ for BP-NOS; the trend of improvement was still continuing in the BP-NOS patients. The number of patients evaluated decreased to $45.9 \%$ (155/338).

Overall trends of the time course changes in HSAS scores were similar to those for HSDS scores, but the scores, in general, were slightly higher than for HSDS. The HSAS scores of the withdrawn patients had hardly changed from the baseline values, although they did not become worse than baseline.

\section{Clinical Global Impressions-Improvement scale scores}

When the patients completed treatment, either at week 52 or at withdrawal, CGI-I scores were evaluated and compared with the baseline scores (Table 3 ). Nearly $60 \%$ of the patients were scored as "very much improved" or "much improved": $63.4 \%$ for BP-I, $74.2 \%$ for BP-II, and $59.8 \%$ for BP-NOS. No BP-II patient was evaluated lower than "no change".

\section{Adherence to LTG treatment}

The time courses of adherence to LTG treatment for all patients and by disease type were plotted using KaplanMeier curves (Figure 1). The adherence curve for all patients $(n=445)$ decreased exponentially. The rate was sustained at $39.6 \%$ from 637 days (1.7 years) onward; no patient in the study withdrew from LTG treatment after 637 days. The longest adherence duration observed was 1,359 days (3.7 years). The median adherence duration (ie, the duration of treatment at $50 \%$ adherence rate), was 399 days (1.1 years); the first $25 \%$ of the patients withdrew within 28 days ( 4 weeks).

For BP-I $(n=41)$, the curve decreased in a stepped pattern. The adherence rate was sustained at $51.1 \%$ from 602 days (1.6 years) onward. The median adherence duration could therefore not be evaluated. The longest adherence duration observed was 1,359 days (3.7 years). The first $25 \%$ of the patients withdrew from LTG treatment within 40 days (5.7 weeks).

For BP-II $(n=66)$, the curve again decreased in a staircase pattern. The adherence rate was sustained at $41.4 \%$ from 637 days ( 1.7 years) onward. The longest adherence duration 
Table 3 Clinical Global Impressions Improvement scale scores at week 52/withdrawal

\begin{tabular}{|c|c|c|c|c|}
\hline Improvement extent ${ }^{\mathbf{a}}$ & All $(n=445), n(\%)$ & BP-I $(n=4 I), n(\%)$ & BP-II (n=66), n (\%) & BP-NOS ( $n=338), n(\%)$ \\
\hline Very much improved & $147(33.03)$ & I7 (4I.46) & $25(37.88)$ & $105(31.07)$ \\
\hline Much improved & $130(29.21)$ & $9(21.95)$ & $24(36.36)$ & $97(28.70)$ \\
\hline Minimally improved & $92(20.67)$ & $9(21.95)$ & $12(18.18)$ & $7 \mathrm{I}(2 \mathrm{I} .0 \mathrm{I})$ \\
\hline No change & $70(15.73)$ & $3(7.32)$ & $5(7.58)$ & $62(18.34)$ \\
\hline Minimally worse & $5(1.12)$ & $3(7.32)$ & $0(0.0)$ & $2(0.59)$ \\
\hline Unknown & I (0.22) & $0(0.0)$ & $0(0.0)$ & I $(0.30)$ \\
\hline
\end{tabular}

Note: aNo subjects were evaluated lower than as "Minimally worse".

Abbreviations: BP-I, bipolar I disorder; BP-II, bipolar II disorder; BP-NOS, bipolar disorder not otherwise specified.

observed was 1,211 days (3.3 years). The median adherence duration was 340 days ( 0.93 years). The first $25 \%$ of patients withdrew within 21 days ( 3 weeks).

For BP-NOS ( $\mathrm{n}=338$ ), the curve decreased exponentially. The adherence rate was sustained at $36.6 \%$ from 586 days (1.60 years) onward. The longest adherence duration observed was 988 days (2.7 years). The median adherence duration was 373 days ( 1.0 year). The first $25 \%$ of the patients withdrew within 28 days (4 weeks). Comparisons between the survival curves of the groups showed the differences were not statistically significant $(P=0.45$, log-rank test) although the curves had quite different appearances.

\section{Time course of concomitant medications}

Time courses of LTG with concomitant medications are indicated in Table $2 \mathrm{~A}$ and $\mathrm{B}$ by classes of psychotropic drug and disease types. At baseline, $88.5 \%(395 / 445)$ of the patients were treated with LTG combined with other drugs.

For the combination of LTG with psychotropic drugs, the patients were administered 3.54 drugs on average during the 52 weeks of the observation period. For the combination with atypical antipsychotics, the number of drugs remained stable for all groups except BP-II (1.09-1.23). For the combination of LTG with ADs, the number of drugs in the BP-I group decreased from 1.43 to 1.11. For the combination of LTG with TA, the subsequent time course differed between the groups (Table 2A). As shown in the time course changes in the mean dose of medications concomitant with LTG (Table 2B), the mean doses of ADs in all patients were gradually increased (177.1 $\mathrm{mg}$ at baseline, $182.2 \mathrm{mg}$ at week 52), while those of atypical antipsychotics were gradually decreased (247.8 $\mathrm{mg}$ at baseline, $224.9 \mathrm{mg}$ at week 52).
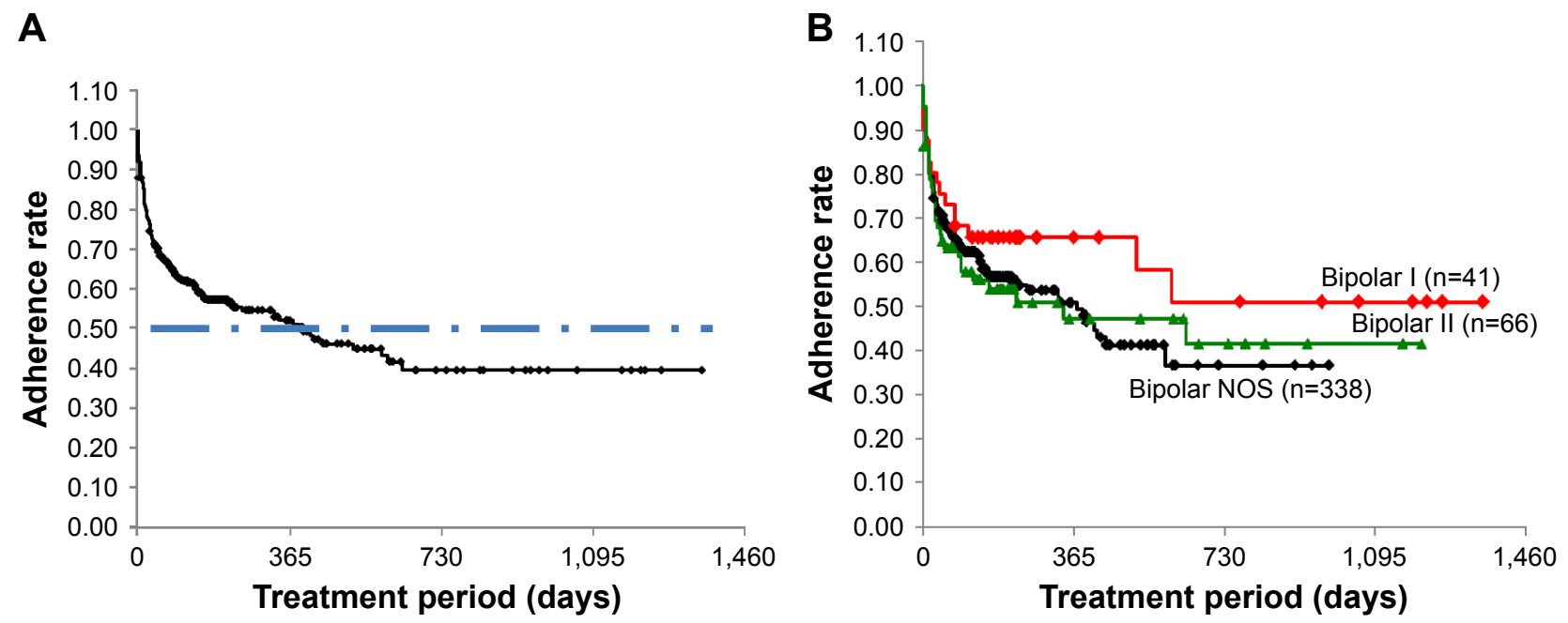

Figure I Time course changes in adherence to lamotrigine treatment.

Notes: Kaplan-Meier curves showing adherence to lamotrigine treatment for all patients $(\mathrm{n}=455 ; \mathbf{A})$ and for each disease type (B). The rate for all patients was sustained at $39.6 \%$ from 637 days ( 1.74 years) onward with a median duration of 399 days ( 1.1 years). The first $25 \%$ of the patients withdrew within 28 days (4 weeks), with no withdrawals after 637 days. The bipolar I rate was sustained at $51.2 \%$ from 602 days ( 1.6 years) onward, and so the median duration could not be calculated; the longest duration was I,359 days ( 3.7 years). The first $25 \%$ of the patients withdrew within 40 days ( 5.7 weeks). The bipolar II rate was sustained at 4 I. $4 \%$ from 637 days ( 1.7 years) onward with a median duration of 340 days ( 0.93 years) and with the longest duration being I, 2 I I days ( 3.3 years). The first $25 \%$ of the patients withdrew within 2 I days ( 3 weeks). The bipolar NOS rate was sustained at $36.6 \%$ from 586 days ( 1.60 years) onward with a median duration of 373 days ( 1.0 year) and the longest duration being 988 days ( 2.7 years). The first $25 \%$ of the patients withdrew within 28 days ( 4 weeks). Although the curves differ considerably in appearance, no statistically significant differences were observed between them $(P=0.45$, log-rank test).

Abbreviation: NOS, not otherwise specified. 
However, no statistical significance was observed in any of these changes (by analysis of variance).

\section{Changes in HSDS and HSAS scores with concomitant medications}

Time course changes in HSDS and HSAS scores with and without ADs are presented in Table 4. The mean HSDS and HSAS scores at baseline were almost the same across the three disease groups, except for the HSAS scores for BP-II, where the mean of 24.26 without ADs was significantly higher than 19.33 with $\operatorname{ADs}(P=0.031)$. At week 24 , the mean HSDS scores for all patients $(15.06$ vs $17.87, P=0.016)$ and BP-NOS (14.95 vs $19.30, P=0.001)$ as well as the HSAS mean score in BP-NOS (14.57 vs $19.67, P=0.002$ ) were significantly lower without ADs than with ADs. Conversely, the mean HSAS score for BP-II was significantly higher without ADs than with ADs (20.69 vs 12.41, $P=0.025)$. At week 52, overall the scores without ADs still tended to be lower than the scores with ADs, but this difference was only significant for the mean HSDS score for all patients (13.75 vs $16.41, P=0.050$ ).

\section{Safety evaluation}

The overall frequencies of adverse events were $22.9 \%$ (104/455) for all patients, 34.1\% (14/41) for BP-I, 22.7\% $(15 / 66)$ for BP-II, and 22.2\% (75/338) for BP-NOS (Table 5). The most common adverse event was skin rash $(22.0 \%$ for BP-I, 16.7\% for BP-II, and 12.1\% for BP-NOS), followed by oral exanthema (1.8\% for all patients) and pharyngalgia (1.1\% for all patients).

\section{Discussion}

The efficacy of LTG was primarily assessed by observing the changes in the patients' self-rated depression and anxiety scores over 1 year or more. An improvement in depression scores was observed generally at week 4 according to the changes in HSDS scores from baseline to week 52 or withdrawal from LTG. The trend of improvement in depression scores continued at week 12 , but then slowed until stabilizing by week 36 . This indicates that LTG could show positive effects within 3 months after the treatment initiation not only for patients with BP-I, but also for those with BP-NOS or BP-II. A previous large-scale study in Japan demonstrated the efficacy of LTG in the prevention of depressive episodes in patients with BP-I; ${ }^{20}$ however, no statistically significant improvement was observed in the BP-I group of this study. A possible reason for this could be the small number of BP-I patients at week $52(\mathrm{n}=22)$ as well as a lower mean HSDS score (indicating less depressive symptoms) at baseline for these patients than for the other disease types $16.5 \pm 6.2$ $(n=22)$. A similar tendency of score decrease was observed in the changes in anxiety symptom scores. Anxiety symptoms improved for the BP-NOS patients, but no notable changes were observed for BP-I and BP-II.

The improved HSDS and HSAS scores were sustained at week 52 (1 year). Adherence to LTG beyond this time was also evaluated where data were available. The median duration of adherence was 399 days, such that just beyond 1 year after the initiation of LTG treatment, $50 \%$ of the patients were still maintaining adherence. For all of the patients, the adherence rate was finally sustained at $39.6 \%$ from 637 days ( 1.7 years), indicating that $\sim 40 \%$ of the patient population with any type of BP treated with LTG could maintain their adherence with good disease condition and tolerability for $>1.7$ years. For BP-I, although the number of patients evaluated was limited $(n=41)$, the adherence rate was sustained at $51.1 \%$ from 602 days ( 1.6 years), indicating that $>50 \%$ of the BP-I patients were able to maintain adherence, showing good disease condition and tolerability for more than a year. The final adherence rate was lowest of all in the BP-NOS group, with $36.6 \%$ of these patients maintaining adherence after 586 days (1.6 years), although $50 \%$ of the patients maintained their adherence for 1 year after LTG treatment. Together, these results suggest that $>50 \%$ of the patients with any type of bipolar disease were able to maintain adherence with good disease condition and tolerability for at least 1 year, demonstrating the long-term efficacy and safety of LTG. The results are also consistent with past reports on LTG tolerability ${ }^{23,34}$ as well as those on efficacy in the prevention of depressive episodes. ${ }^{17,23,35}$ However, $25 \%$ of the patients in the study withdrew within the first 42 days (21 days for BP-II, 28 days for all patients and for BP-NOS, and 40 days for BP-I as a quartile), for various reasons. Despite the different appearances of the final adherence rate (higher in BP-I and lowest in BP-NOS), there were no statistically significant differences among the BP groups. As shown in Table 1 (age at initial visit/onset), however, the disease duration in BP-I is $\sim 3$ years longer than that in BP-II and BP-NOS. According to one-way analysis of variance, the disease duration among the three BP groups was statistically significant $(P=0.0284)$. Moreover, statistically significant differences $(P=0.0285)$ were observed between BP-I (8.3 years) and BP-NOS (5.7 years) with the mean difference of 2.83 years by Scheffe's multiple pairwise comparison. We assume that this difference in the disease duration could have enhanced the insight into disease in the BP-I patients, resulting in better adherence. 


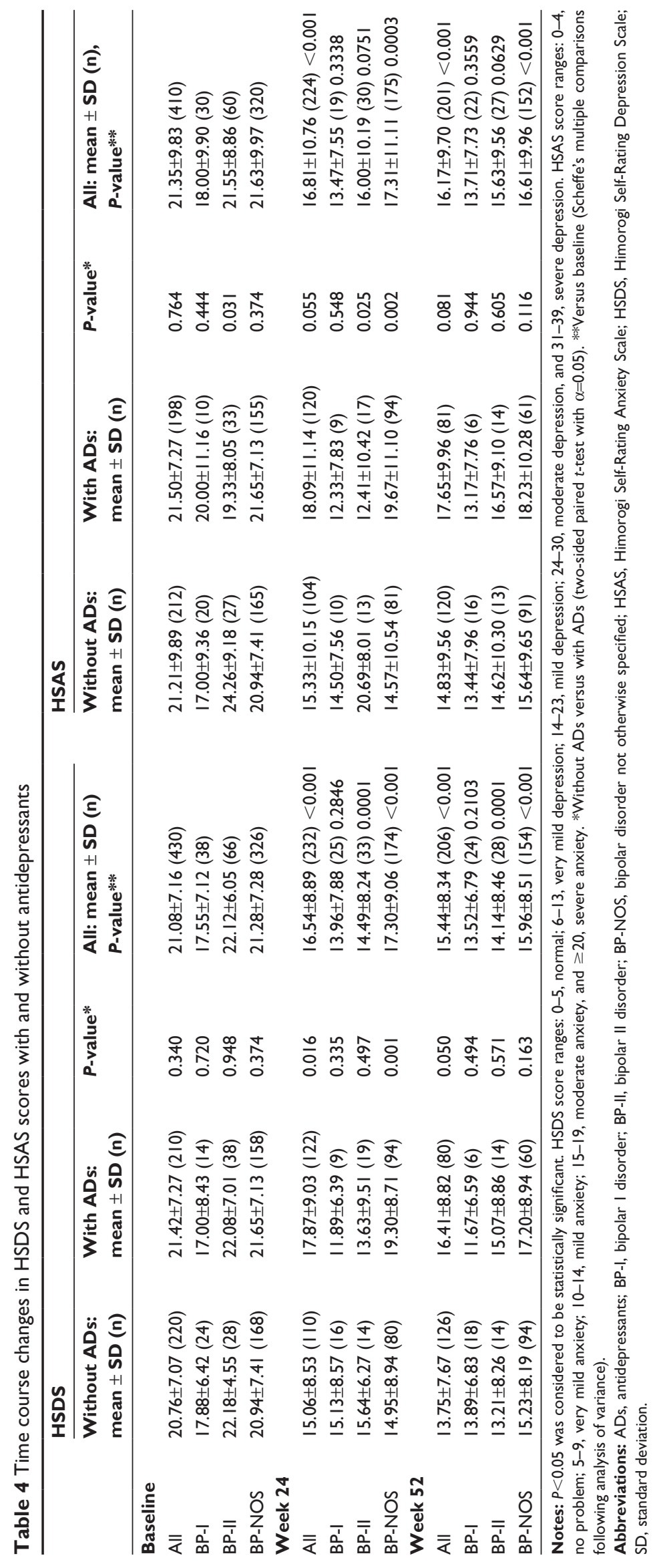


Table 5 Adverse events experienced by the patients

\begin{tabular}{|c|c|c|c|c|}
\hline Adverse event & All $(n=445), n(\%)$ & BP-I (n=4I), n (\%) & BP-II (n=66), n (\%) & BP-NOS $(n=338), n(\%)$ \\
\hline Total & $104(22.86)$ & $14(34.15)$ & $15(22.73)$ & $75(22.19)$ \\
\hline Skin rash & $61(13.7)$ & $9(22.0)$ & II (I6.7) & $4 I(12.1)$ \\
\hline Oral exanthema & $8(1.8)$ & I (2.4) & I (I.5) & $6(1.8)$ \\
\hline Pharyngalgia & $5(I .1)$ & I (2.4) & & $4(1.2)$ \\
\hline Lymph node swelling & $4(0.9)$ & & & $4(1.2)$ \\
\hline Conjunctivitis & $4(0.9)$ & $2(4.9)$ & & $2(0.6)$ \\
\hline Nausea & $3(0.7)$ & & & $3(0.9)$ \\
\hline Impatience & $3(0.7)$ & & & $3(0.9)$ \\
\hline Dizziness & $2(0.4)$ & & & $2(0.6)$ \\
\hline Feel down & $2(0.4)$ & & & $2(0.6)$ \\
\hline Fever & $2(0.4)$ & & & $2(0.6)$ \\
\hline Fatigue & $2(0.4)$ & & $\mathrm{I}(\mathrm{I} .5)$ & I $(0.3)$ \\
\hline Diarrhea & $\mathrm{I}(0.2)$ & & & $\mathrm{I}(0.3)$ \\
\hline Feeling of cerebral atrophy & I $(0.2)$ & & & $\mathrm{I}(0.3)$ \\
\hline Anhedonia & $\mathrm{I}(0.2)$ & & & $\mathrm{I}(0.3)$ \\
\hline Insomnia & $\mathrm{I}(0.2)$ & & & $\mathrm{I}(0.3)$ \\
\hline Panic attack & $\mathrm{I}(0.2)$ & & & I (0.3) \\
\hline Suicide ideation & $\mathrm{I}(0.2)$ & I (2.4) & & \\
\hline Drowsiness & $\mathrm{I}(0.2)$ & & $\mathrm{I}(\mathrm{I} .5)$ & \\
\hline Acute pancreatitis & $\mathrm{I}(0.2)$ & & I (I.5) & \\
\hline
\end{tabular}

Abbreviations: BP, bipolar disorder; BP-I, bipolar I disorder; BP-Il, bipolar II disorder; BP-NOS, bipolar disorder not otherwise specified.

Among the patients who underwent combination therapy, $87.6 \%$ were administered LTG plus psychotropic drugs with an average of 3.54 drugs per patient (range: 1-15), with the rest of the patients (12.4\%: 49/394) treated by LTG monotherapy. Although the average number of concomitant drugs per patient was stable during the study period, the rate of multidrug therapy gradually decreased (except in the BP-I group) from baseline toward week 52 (all patients, 87.6\%-81.8\% [-6.5\%]; BP-II, $100 \%-93.3 \%[-6.7 \%]$; BP-NOS, $85.0 \%-78.8 \%$ [-6.2\%]); but BP-I ( $85.4 \%-87.5 \%$ [2.1\%]), which meant that $6 \%-7 \%$ of the patients with combination therapy transferred to LTG monotherapy, showing improvement in the CGI-I and HSDS/HSAS scores.

These results suggest that reducing concomitant psychotropic agents, especially ADs, may not affect the efficacy of LTG, which implies LTG monotherapy potentially has sufficient efficacy for the long-term treatment of BP. ADs may be effective for short periods with or without mood stabilizers in depressed bipolar patients. ${ }^{4}$ However, the use of ADs is not to be recommended for long-term treatment in clinical practice as ADs may be associated with the development of rapid cycling during the course of the disease. ${ }^{11,36-38}$ In this study, a total of 31 out of 445 bipolar patients indicated cycle accelerations. Moreover, rapid cycling may occur differently depending on the types of BP. The highest incidence rate of cycle acceleration was observed in BP-I $(38.1 \% ; 16 / 42)$ followed by BP-II $(22.7 \%$; $15 / 66$ ), while no cycle acceleration appeared in BP-NOS
$(0 \% ; 0 / 337)$, resulting in a statistically significant difference $(P<0.001$; chi-square test $)$.

Furthermore, ADs lose their effectiveness and may actually induce depression with long-term use, ${ }^{39}$ and more depressive episode criteria was predicted in those who continued $\mathrm{AD}$ treatment as opposed to discontinued $\mathrm{AD}$ treatment. ${ }^{40}$

Interestingly, the efficacy of AD combination therapy differed between the disease types. The mean depression and anxiety scores of the BP-I patients showed more severe symptoms in the patients without ADs than in those with ADs, whereas in BP-NOS and BP-II symptoms were less severe without ADs than with ADs, although the means at baseline were almost the same. However, whether this result was caused by greater AD use or was simply a reflection of attempts at treating a more difficult course of illness remains to be investigated further.

Concurrent anxiety was assessed using a self-rating anxiety scale, ${ }^{25}$ which is employed in daily clinical practice at the study sites as anxiety symptom severity could be an important reason for treatment nonresponse. People with BP have depressive symptoms for a substantial proportion of the time $^{4,5,41}$ and anxiety often co-occurs with the depression, ${ }^{12,42}$ resulting in long-term nonresponse. ${ }^{13}$ Our study results showed that anxiety symptoms improved only slightly in patients both with combination therapy with ADs and with LTG monotherapy, suggesting that the use of ADs may not affect the improvement of anxiety symptoms in bipolar patients. Although we mainly focused on anxiety symptoms 
and ADs as the most relevant predictors of nonadherence among the patients, when treating BP, it would be important to consider other relevant factors of nonadherence, such as younger age, comorbidity with substance use and personality disorders, patients' beliefs, poor insight, illness severity, treatment-related side effects, specific features of the disease, and a poor therapeutic alliance, as suggested by Pompili et al. ${ }^{14}$

The safety of LTG was assessed by examining adverse events and their frequencies. The overall frequency of adverse events in our study was $22.9 \%$ (104/455), with the most common adverse event being skin rash $(13.7 \%$; 61/445). The incidence of benign skin reactions in this study was higher than that observed in previous studies: between $8 \%$ and $11 \%$ by Ketter et $\mathrm{al}^{43}$ and $12.7 \%$ by Woo et al. ${ }^{44} \mathrm{~A}$ recent meta-analysis showed an even lower rate of $7.2 \%(214 / 2,977$ patients) in retrospective studies. ${ }^{45}$ The reason for this result could be the particular attention we paid to the onset of rashes; patients with the slightest sign of rash were withdrawn immediately at a physician's discretion. In addition, careful dose titration was performed so that no severe or life-threatening rashes would occur. Concomitant therapy with valproic acid (VPA), which has a well-recognized association with the incidence of rash, was also examined. At baseline, the concomitant ratios of VPA were 24.0\% (107/445), 26.8\% (11/41), 37.9\% (25/66), and $21.1 \%(71 / 336)$ for all patients, BP-I, B-II, and BP-NOS, respectively. However, the frequencies of skin rash were comparable between the patients administered VPA and those who were not across all the disease types, which may have been the result of the careful titration and particular attention on the incidence of skin rashes. Although no statistical significance was observed in our study, the incidence of skin rash may vary depending on the disease types, being greater in the types where symptoms of mania and burden of illness appear more clearly: $12.1 \%$ for BP-NOS, $16.7 \%$ for BP-II, and $22.0 \%$ for BP-I. However, a clear reason for this phenomenon cannot be clarified at this point. A possible hypothesis could be that both the central nervous system and skin cells originate in ectoderms, ${ }^{46}$ which may have a common mechanism that affects each other. In a study that investigated comorbid disorders and compared their prevalence in hospitalizations of persons with or without BP, higher proportions of most psychiatric and some general medical conditions including various diseases of the skin and subcutaneous tissue (proportional morbidity ratio $=2.6-4.2$ ) were shown. ${ }^{47}$ Further investigations regarding this factor are needed.

There are several limitations that should be considered when interpreting the results of our study. 1) There was no control group, and so objective comparisons were not possible. 2) HSDS and HSAS are self-rating scales and thus lack objectivity. 3) The data were drawn from an observational study, which could lead to recall bias in some cases. 4) The number of patients in BP-I (41, 9.2\%) and BP-II (66, 14.8\%) groups was smaller compared to BP-NOS (338, 76.0\%), which should not be considered as predictive of larger groups' results. Finally, patients with psychiatric disorders tend to be treated with multidrug therapy in Japan, which results in the potential for confounding effects of the multiple drugs. This cultural background may have been reflected in the results of our study, which should be taken into consideration when examining the results of this study.

Nevertheless, we were able to establish that $50 \%$ of the patients with any type of BP could be treated with LTG for at least 1 year and $\sim 40 \%$ for $>1.5$ years when careful and adequate titration was provided. Among the three disease groups, the patients with BP-NOS, who formed the majority of our study population (reflecting the worldwide prevalence of this disease type among BPs), responded particularly well to long-term LTG treatment, which could be one of the reasons for the favorable results of our study. This findings also suggest that bipolar symptoms treated with LTG can improve with or without the use of ADs, with the efficacy of ADs differing between disease types. This result highlights the need for further verification of the efficacy of $\mathrm{AD}$ in bipolar treatment in large-scale prospective studies and clinical trials.

\section{Acknowledgments}

The authors thank Mr Kenichiro Tsumura for his technical assistance on data analysis and manuscript preparation. They also thank Enago (www.enago.jp) for the review of English language. This study was supported by funding from GlaxoSmithKline. The sponsor has no involvement in the study design, data analysis, or manuscript preparation. The views expressed in this manuscript are those of the authors. YW has received speaker's honoraria from Pfizer, GlaxoSmithKline, Otsuka Pharmaceutical, Janssen Pharmaceutical, Meiji, Eli Lilly Japan, MSD, and Astellas Pharma. SH has received speaker's honoraria from Meiji Seika Pharma, Janssen Pharmaceutical, Eli Lilly Japan, GlaxoSmithKline, Chugai Pharma, Mochida Pharmaceutical, Otsuka Holdings, and Dainippon Sumitomo Pharma.

\section{Disclosure}

This study was funded by GlaxoSmithKline. The authors report no other conflicts of interest in this work. 


\section{References}

1. American Psychiatric Association. Diagnostic and Statistical Manual of Mental Disorders. 5th ed. (DSM-5). Arlington, Texas: American Psychiatric Publishing; 2013.

2. Merikangas KR, Jin R, He JP, et al. Prevalence and correlates of bipolar spectrum disorder in the world mental health survey initiative. Arch Gen Psychiatry. 2011;68(3):241-251.

3. Etain B, Lajnef M, Bellivier F, et al. Clinical expression of bipolar disorder type I as a function of age and polarity at onset: convergent findings in samples from France and the United States. J Clin Psychiatry. 2012;73(4):561-566.

4. Tondo L, Baldessarini RJ, Vázquez G, Lepri B, Visioli C. Clinical responses to antidepressants among 1036 acutely depressed patients with bipolar or unipolar major affective disorders. Acta Psychiatr Scand. 2013;127(5):355-364.

5. Undurraga J, Baldessarini RJ, Valentí M, et al. Bipolar depression: clinical correlates of receiving antidepressants. J Affect Disord. 2012;139(1):89-93.

6. Baldessarini RJ, Leahy L, Arcona S, Gause D, Zhang W, Hennen J. Patterns of psychotropic drug prescription for U.S. patients with diagnoses of bipolar disorders. Psychiatr Serv. 2007;58(1):85-91.

7. Baldessarini R, Henk H, Sklar A, Chang J, Leahy L. Psychotropic medications for patients with bipolar disorder in the United States: polytherapy and adherence. Psychiatr Serv. 2008;59(10):1175-1183.

8. Sussman M, Friedman M, Korn JR, Hassan M, Kim J, Menzin J. The relationship between use of antidepressants and resource utilization among patients with manic or mixed bipolar disorder episodes: findings from a managed care setting. J Affect Disord. 2012;138(3):425-432.

9. Altshuler LL, Post RM, Leverich GS, Mikalauskas K, Rosoff A, Ackerman L. Antidepressant-induced mania and cycle acceleration: a controversy revisited. Am J Psychiatry. 1995;152(8):1130-1138.

10. Ghaemi SN, Wingo AP, Filkowski MA, Baldessarini RJ. Long-term antidepressant treatment in bipolar disorder: meta-analyses of benefits and risks. Acta Psychiatr Scand. 2008;118(5):347-356.

11. Valentí M, Pacchiarotti I, Undurraga J, et al. Risk factors for rapid cycling in bipolar disorder. Bipolar Disord. 2015;17(5):549-559.

12. Pavlova B, Perlis RH, Alda M, Uher R. Lifetime prevalence of anxiety disorders in people with bipolar disorder: a systematic review and meta-analysis. Lancet Psychiatry. 2015;2(8):710-717.

13. Post RM, Leverich GS, Altshuler LL, et al. Relationship of prior antidepressant exposure to long-term prospective outcome in bipolar I disorder outpatients. J Clin Psychiatry. 2012;73(7):924-930.

14. Pompili M, Venturini P, Palermo M, et al. Mood disorders medications: predictors of nonadherence - review of the current literature. Expert Rev Neurother. 2013;13(7):809-825.

15. Murru A, Pacchiarotti I, Nivoli AM, et al. Rates and clinical correlates of treatment non-adherence in schizoaffective bipolar patients. Acta Psychiatr Scand. 2012;125(5):412-418.

16. Montes JM, Maurino J, de Dios C, Medina E. Suboptimal treatment adherence in bipolar disorder: impact on clinical outcomes and functioning. Patient Prefer Adherence. 2013;7:89-94.

17. Reid JG, Gitlin MJ, Altshuler LL. Lamotrigine in psychiatric disorders. J Clin Psychiatry. 2013;74(7):675-684

18. Weisler RH, Calabrese JR, Bowden CL, Ascher JA, DeVeaugh-Geiss J, Evoniuk G. Discovery and development of lamotrigine for bipolar disorder: a story of serendipity, clinical observations, risk taking, and persistence. J Affect Disord. 2008;108(1-2):1-9.

19. Goodwin GM, Bowden CL, Calabrese JR, et al. A pooled analysis of 2 placebo-controlled 18-month trials of lamotrigine and lithium maintenance in bipolar I disorder. J Clin Psychiatry. 2004;65(3):432-441.

20. Koyama T, Higuchi T, Yamawaki N, et al. Study SCA 104779, an evaluation of BW430C (lamotrigine) versus placebo in the prevention of mood episodes in bipolar I disorder patients. Japanese J Clin Psychiatry. 2011;40(3):369-383. Japanese.

21. Calabrese JR, Sullivan JR, Bowden CL, et al. Rash in multicenter trials of lamotrigine in mood disorders: clinical relevance and management. J Clin Psychiatry. 2002;63(11):1012-1019.
22. Mockenhaupt M, Messenheimer J, Tennis P, Schlingmann J. Risk of Stevens-Johnson syndrome and toxic epidermal necrolysis in new users of antiepileptics. Neurology. 2005;64(7):1134-1138.

23. Miura T, Noma H, Furukawa TA, et al. Comparative efficacy and tolerability of pharmacological treatments in the maintenance treatment of bipolar disorder: a systematic review and network meta-analysis. Lancet Psychiatry. 2014;1(5):351-359.

24. Mimura C, Murashige M, Oda T, Watanabe Y. Development and psychometric evaluation of a Japanese scale to assess depression severity: himorogi self-rating depression scale. Int J Psychiatry Clin Pract. 2011;15(1):50-55.

25. Mimura C, Nishioka M, Sato N, Hasegawa R, Horikoshi R, Watanabe Y. A Japanese scale to assess anxiety severity: development and psychometric evaluation. Int J Psychiatry Med. 2011; 41(1):29-45.

26. Guy W. ECDEU assessment manual fro psychopharmacology: publication ADM 76-338. Clinical Global Impressions (CGI) Scale. Washington, DC: Department of Health, Education, and Welfare; 1976: $218-222$.

27. Hamilton M. The assessment of anxiety states by rating. $\mathrm{Br} J \mathrm{Med}$ Psychol. 1959;32(1):50-55.

28. Williams JB. A structured interview guide for hamilton depression rating scale. Archi Gen Psychiatry. 1988;45(8):742-747.

29. Lin EH, Von Korff M, Katon W, et al. The role of the primary care physician in patients' adherence to antidepressant therapy. Med Care. 1995;33(1):67-74.

30. Busner J, Targum SD. The clinical global impressions scale: applying a research tool in clinical practice. Psychiatry (Edgmont). 2007;4(7): 28-37.

31. Spielmans GI, McFall JP. A comparative meta-analysis of Clinical Global Impressions change in antidepressant trials. $J$ Nerv Ment Dis. 2006; 194(11):845-852.

32. Leucht S, Engel RR. The relative sensitivity of the clinical global impressions scale and the brief psychiatric rating scale in antipsychotic drug trials. Neuropsychopharmacology. 2006;31(2):406-412.

33. Zaider TI, Heimberg RG, Fresco DM, Schneier FR, Liebowitz MR. Evaluation of the clinical global impression scale among individuals with social anxiety disorder. Psychol Med. 2003;33(4):611-622.

34. Seo HJ, Chiesa A, Lee SJ, et al. Safety and tolerability of lamotrigine: results from 12 placebo-controlled clinical trials and clinical implications. Clin Neuropharmacol. 2011;34(1):39-47.

35. Grande I, Balanzá-Martínez V, Jiménez-Arriero M, et al; SIN-DEPRES Group. Clinical factors leading to lamotrigine prescription in bipolar outpatients: subanalysis of the SIN-DEPRES study. J Affect Disord. 2012;143(1-3):102-108.

36. Baldessarini RJ, Tondo L, Floris G, Hennen J. Effects of rapid cycling on response to lithium maintenance treatment in 360 bipolar I and II disorder patients. J Affect Disord. 2000;61(1-2):13-22.

37. Bauer M, Beaulieu S, Dunner DL, Lafer B, Kupka R. Rapid cycling bipolar disorder-diagnostic concepts. Bipolar Disord. 2008;10(1 Pt 2): 153-162.

38. Calabrese JR, Shelton MD, Rapport DJ, Kujawa M, Kimmel SE, Caban S. Current research on rapid cycling bipolar disorder and its treatment. $J$ Affect Disord. 2001;67(1-3):241-255.

39. El-Mallakh RS, Gao Y, Jeannie Roberts R. Tardive dysphoria: the role of long term antidepressant use in-inducing chronic depression. Med Hypotheses. 2011;76(6):769-773.

40. El-Mallakh RS, Vöhringer PA, Ostacher MM, et al. Antidepressants worsen rapid-cycling course in bipolar depression: a STEP-BD randomized clinical trial. $J$ Affect Disord. 2015;184:318-321.

41. Judd LL, Schettler PJ, Akiskal HS, et al. Long-term symptomatic status of bipolar I vs bipolar II disorders. Int $J$ Neuropsychopharmacol. 2003;6(2):127-137.

42. Kessler RC, Chiu WT, Demler O, Merikangas KR, Walters EE. Prevalence, severity, and comorbidity of 12-month DSM-IV disorders in the National Comorbidity Survey Replication. Arch Gen Psychiatry. 2005; 62(6):617-627. 
43. Ketter TA, Wang PW, Chandler RA, et al. Dermatology precautions and slower titration yield low incidence of lamotrigine treatment-emergent rash. J Clin Psychiatry. 2005;66(5):642-645.

44. Woo YS, Bahk WM, Jon DI, et al. Rash in adult patients receiving lamotrigine to treat bipolar I disorder in Korea: a multicenter, prospective, naturalistic, open-label trial. Prog Neuropsychopharmacol Biol Psychiatry. 2009;33(7):1147-1152.

45. Wang XQ, Xiong J, Xu WH, et al. Risk of a lamotrigine-related skin rash: current meta-analysis and postmarketing cohort analysis. Seizure. 2015;25:52-61.
46. Slack JMW. Essential Developmental Biology. 3rd ed. Wiley-Blackwell; 2012.

47. Weber NS, Fisher JA, Cowan DN, Niebuhr DW. Psychiatric and general medical conditions comorbid with bipolar disorder in the National Hospital Discharge Survey. Psychiatr Serv. 2011;62(10):1152-1158.

\section{Publish your work in this journal}

Neuropsychiatric Disease and Treatment is an international, peerreviewed journal of clinical therapeutics and pharmacology focusing on concise rapid reporting of clinical or pre-clinical studies on a range of neuropsychiatric and neurological disorders. This journal is indexed on PubMed Central, the 'PsycINFO' database and CAS, and is the official journal of The International Neuropsychiatric Association (INA). The manuscript management system is completely online and includes a very quick and fair peer-review system, which is all easy to use. Visit http://www.dovepress.com/testimonials.php to read real quotes from published authors.

Submit your manuscript here: http://www.dovepress.com/neuropsychiatric-disease-and-treatment-journal 